\title{
Prognostic value of circulating amino-terminal pro-C-type natriuretic peptide in critically ill patients
}

Alexander Koch, Sebastian Voigt, Edouard Sanson, Hanna Dückers, Andreas Horn, Henning W Zimmermann, Christian Trautwein, Frank Tacke*

\begin{abstract}
Introduction: C-type natriuretic peptide (CNP) is a paracrine molecule which is mainly synthesized in the vasculature. High levels have been reported in sepsis, and CNP has been proposed as a biomarker predicting sepsis in traumatized patients. We aimed at evaluating the diagnostic and prognostic value of N-terminal pro-CNP (NTproCNP) for predicting sepsis, disease severity and mortality in critically ill medical patients.

Methods: 273 critically ill patients (197 patients with sepsis or septic shock, 76 without evidence of sepsis) and 43 healthy controls were consecutively included in a prospective clinical single-center non-interventional study at the Medical Intensive Care Unit, RWTH-University Aachen, Germany. Patients' outcome was followed for about 1 year. NT-proCNP serum concentrations were determined upon ICU admission, as well as in the mornings of day 3 and day 7 after admission. Intensive care treatment measures as well as routine and experimental laboratory parameters were recorded and analyzed.
\end{abstract}

Results: NT-proCNP serum concentrations upon admission to the ICU were elevated in critically ill patients as compared with healthy controls. Patients with sepsis had significantly higher NT-proCNP levels than non-sepsis patients. NT-proCNP was strongly associated with inflammatory parameters (i.e. C-reactive protein, procalcitonin and TNF- $\alpha$ ), biomarkers of organ dysfunction and clinical composite scores (APACHE-II, SOFA, SAPS2). NT-proCNP levels at admission and day 3 were found to be a strong predictive marker for ICU- and overall survival. Moreover, a decline of serum NT-proCNP after admission to the ICU was associated with reduced mortality. The predictive power of serum NT-proCNP was similar to 'conventional' prognostic tools such as clinical scores.

Conclusions: NT-proCNP is significantly elevated in critically ill patients, with highest levels in sepsis. Inflammation as well as organ function are strongly associated with NT-proCNP serum concentrations. Low initial NT-proCNP levels and a decline during initial treatment indicate a favourable ICU- and long-term outcome.

\section{Introduction}

The natriuretic peptide family consists of three distinctive members: atrial natriuretic peptide (ANP), brain natriuretic peptide (BNP) and C-type natriuretic peptide (CNP). These peptides exert multiple potent diuretic, natriuretic and vasorelaxant functions, thereby directly influencing body-fluid homeostasis and blood pressure control [1,2]. As ANP and BNP are mainly derived from the heart in response to atrial and ventricular stretching,

\footnotetext{
* Correspondence: frank.tacke@gmx.net

Department of Medicine III, RWTH-University Hospital Aachen, Pauwelsstrasse 30, 52074 Aachen, Germany
}

they have been thought to act as cardiac hormones and linked to cardiac dysfunction [2,3]. In patients with severe sepsis, BNP has been proposed as a useful biomarker to predict survival $[4,5]$, most likely by indicating septic myocardial depression $[4,5]$.

CNP is synthesized as a precursor proCNP protein, and conversion of proCNP to the biologically active hormone CNP is processed by the intracellular endoprotease furin [6]. Amino-terminal pro-C-type natriuretic peptide (NT-proCNP) is the N-terminal fragment of the C-type natriuretic peptide precursor. As a cleavage product of proCNP, NT-proCNP circulates in equimolar
C Biomed Central

(c) 2011 Koch et al.; licensee BioMed Central Ltd. This is an open access article distributed under the terms of the Creative Commons Attribution License (http://creativecommons.org/licenses/by/2.0), which permits unrestricted use, distribution, and reproduction in any medium, provided the original work is properly cited. 
amounts with CNP in human plasma and is considered to be a more reliable marker of the extent of CNP biosynthesis [7]. Due to its extra-cardiac origin and its high expression in the brain, CNP was initially believed to be a neuropeptide, involved in central regulatory mechanisms [8,9]. At present it is known that CNP is widely expressed in various tissues, with particularly high concentrations in the vascular endothelium [10] and chondrocytes [11], inducing vasorelaxation and vascular remodeling, as well as regulating bone growth [12]. Compared with ANP and BNP, CNP exerts limited diuretic and natriuretic functions, but counteracts angiotensin II- or endothelin-1-induced vasoconstriction and complements the actions of other endothelial vasorelaxant mediators such as nitric oxide (NO) and prostacyclin [13]. IL-1, endotoxins and particularly TNF- $\alpha$, which are increased in states of sepsis, can stimulate CNP release from isolated endothelial cells and in this way regulate local vascular tone [14]. CNP release in response to proinflammatory cytokines suggests an interaction of macrophageal cytokine synthesis and vascular endothelium [15]. This link indicates a potential pathophysiological role of CNP in sepsis and septic shock, which are characterized by arteriolar vasodilatation, hypotension, and inadequate tissue perfusion [16]. In a small cohort of patients with sepsis and septic shock, high serum CNP concentrations have been demonstrated [17]. Moreover, in a recent study, NTproCNP has been proposed as a novel biomarker for predicting the development of sepsis in multiple trauma patients [18]. The diagnostic and prognostic value of NT-proCNP measurements in critically ill medical patients is currently unknown.

We therefore conducted a large study with critically ill patients in a medical ICU, performing longitudinal measurements of NT-proCNP serum concentrations during the first week of ICU treatment, to address whether NT-proCNP is activated in critical illness, whether NTproCNP has diagnostic value for sepsis and/or multiorgan failure, and whether NT-proCNP can serve as a prognostic predictor for ICU and long-term survival.

\section{Materials and methods}

\section{Study design and patient characteristics}

The study protocol was conducted in accordance with the ethical standards laid down in the Declaration of Helsinki and approved by the local ethics committee (ethics committee of the University Hospital Aachen, RWTH-University, Aachen, Germany, reference number EK 150/06). We investigated 273 patients (172 male, 101 female with a median age of 64 years; range 18 to 90 years) who were admitted consecutively to the General Internal Medicine ICU at the RWTH-University Hospital Aachen, Germany (Table 1). Written informed
Table 1 Disease etiology of the study population

\begin{tabular}{lcc}
\hline & Sepsis & Non-sepsis \\
\hline & $n=197$ & $n=76$ \\
$\begin{array}{l}\text { Etiology of sepsis critical illness } \\
\text { Site of infection } \mathrm{n}(\%)\end{array}$ & \\
$\quad$ pulmonary & $117(60 \%)$ & \\
$\quad$ abdominal & $30(15 \%)$ & \\
$\quad$ urogenital & $10(5 \%)$ & \\
$\quad$ others & $40(20 \%)$ & \\
$\begin{array}{l}\text { Etiology of non-sepsis critical illness } \\
\mathrm{n}(\%)\end{array}$ & & $19(25 \%)$ \\
$\quad$ decompensated liver cirrhosis & & $25(33 \%)$ \\
$\quad$ cardio-pulmonary disease & & \\
$\quad$ others & & \\
\hline
\end{tabular}

consent was obtained from the patient, his or her spouse, or the appointed legal guardian. Patients that were expected to have a short-term $(<72$ hours) intensive care treatment due to post-interventional observation or acute intoxication were not included in this study [19]. Medium length of stay at the ICU was nine days (range 1 to 137 days) and medium length of stay in hospital was 27 days (range 2 to 151 days).

We prospectively collected patient data, clinical information and blood samples. The clinical course of patients was observed in a follow-up period by directly contacting the patients, the patients' relatives, or their primary care physicians. Critical care patients were divided upon ICU admission into two categories: sepsis patients and non-sepsis patients. Patients in the sepsis group met the criteria proposed by the American College of Chest Physicians and the Society of Critical Care Medicine Consensus Conference Committee for severe sepsis and septic shock [20].

The control group consisted of 43 healthy blood donors ( 28 male, 15 female; median age 53 years, range 24 to 68 years) from the local blood transfusion institute at the University Hospital Aachen. At our blood transfusion institute, all volunteers that donate blood agreed (after informed consent) to contribute to ongoing biomarker studies. All control subjects had normal values for blood counts, $\mathrm{C}$-reactive protein, and liver enzymes, and tested negative for hepatitis B and C and HIV.

In addition, ICU patients were divided according to their Acute Physiology and Chronic Health Evaluation (APACHE) II score into patients with "moderate disease severity" (APACHE-II < 10, with a statistical risk of death below 10\%) and "high disease severity" (APACHEII > 10) [21].

\section{Characteristics of sepsis and non-sepsis patients}

Among the 273 critically ill patients enrolled in this study, 197 patients conformed to the criteria of bacterial 
sepsis (Table 1). Non-sepsis patients were admitted to the ICU mainly due to cardiopulmonary diseases (myocardial infarction, pulmonary embolism, and cardiac pulmonary edema), decompensated liver cirrhosis, or other critical conditions, and did not differ in age or sex from sepsis patients (Table 1). Compared with the cohort of non-sepsis patients, sepsis patients were more often in need of mechanical ventilation in the longer term (Table 2) and displayed significantly higher levels of routinely used biomarkers of inflammation (i.e. C-reactive protein, procalcitonin, white blood cell count; data not shown). Both groups did not differ in APACHE-II score and simplified acute physiology score (SAPS) 2, vasopressor demand, or laboratory parameters indicating liver or renal dysfunction (data not shown).

\section{NT-proCNP measurements}

Prior to therapeutic interventions, blood samples were collected upon admission to the ICU, as well as in the morning of day three and seven after admission. Following centrifugation at $2000 \mathrm{~g}$ at $4^{\circ} \mathrm{C}$ for 10 minutes, serum and plasma aliquots of $1 \mathrm{~mL}$ were frozen immediately at $-80^{\circ} \mathrm{C}$. NT-proCNP serum concentrations were analyzed using a specific commercial enzyme immunoassay (\#BI-20872, BioMedica, Vienna, Austria; distributor: Immundiagnostik AG, Bensheim, Germany). Intra-assay coefficient of variation was 5.3 to $8.3 \%$, and inter-assay coefficient of variation was 7 to $9 \%$. IL-6, IL10, TNF- $\alpha$ (all Siemens Healthcare, Erlangen, Germany), and procalcitonin (Kryptor, B.R.A.H.M.S. Diagnostica, Henningsdorf, Germany) were measured by commercial chemiluminescence assays, following manufacturers' instructions.

\section{Statistical analysis}

Data are given as median and range due to the skewed distribution of most of the parameters. Differences between two groups were assessed by Mann-Whitney$U$-test and multiple comparisons between more than two groups have been conducted by Kruskal-Wallis analysis of variance and Mann-Whitney- $U$-test for post hoc analysis. Box plot graphics illustrate comparisons between subgroups and they display a statistical summary of the median, quartiles, range, and extreme values. The whiskers extend from the minimum to the maximum value excluding outside and far out values, which are displayed as separate points. An outside value (indicated by an open circle) was defined as a value that is smaller than the lower quartile minus 1.5-times interquartile range, or larger than the upper quartile plus 1.5-times the interquartile range. A far out value (indicated by an asterisk) was defined as a value that is smaller than the lower quartile minus three times interquartile range, or larger than the upper quartile

Table 2 Baseline patient characteristics and NT-proCNP serum concentrations

\begin{tabular}{|c|c|c|c|}
\hline Parameter & All patients & Sepsis & Non-sepsis \\
\hline Number & 273 & 197 & 76 \\
\hline Sex (male/female) & $172 / 101$ & $128 / 69$ & $44 / 32$ \\
\hline Age median (range) (years) & $64(18-90)$ & $65(20-90)$ & $60(18-85)$ \\
\hline APACHE-II score median (range) & $17(2-40)$ & $18(3-40)$ & $15(2-31)$ \\
\hline SAPS2 score median (range) & $44(0-80)$ & $44.5(0-79)$ & $41.5(13-80)$ \\
\hline ICU days median (range) & $9(0-137)$ & $12 * *(0-137)$ & $6 *(1-45)$ \\
\hline Hospital days median (range) & $27(2-151)$ & $30 * *(2-151)$ & $14 * *(2-85)$ \\
\hline $\begin{array}{l}\text { Death during ICU } \\
\mathrm{n}(\%)\end{array}$ & $76(27.8 \%)$ & $61(31.0 \%)$ & $15(19.7 \%)$ \\
\hline Death during follow up n (\%) & $132(50.2 \%)$ & $101(53.2 \%)$ & $31(42.5 \%)$ \\
\hline Mechanical ventilation n (\%) & $194(73.2 \%)$ & $144(75 \%)$ & $50(68.5 \%)$ \\
\hline Ventilation time median (range) (hours) & $126(0-2966)$ & $180 *(0-2966)$ & $48.5(0-986)$ \\
\hline Pre-existing diabetes n (\%) & $88(33.1 \%)$ & $60(31.3 \%)$ & $28(37.8 \%)$ \\
\hline BMI median (range) $\left(\mathrm{m}^{2} / \mathrm{kg}\right)$ & $25.8(14.0-66.7)$ & $25.9(14.0-66.7)$ & $25.8(15.9-53.3)$ \\
\hline $\begin{array}{l}\text { NT-proCNP day } 1 \text { median (range) } \\
\text { (pmol/L) }\end{array}$ & $4.07(0-42)$ & $5.6(0-42) * *$ & $1.48(0-42) * *$ \\
\hline $\begin{array}{l}\text { NT-proCNP day } 3 \text { median (range) } \\
\text { (pmol/L) }\end{array}$ & $4.79(0-42)$ & $5.81(0-42) *$ & $0.90(0-42) *$ \\
\hline $\begin{array}{l}\text { NT-proCNP day } 7 \text { median (range) } \\
\text { (pmol/L) }\end{array}$ & $3.91(0-42)$ & $4.59(0-42)$ & $2.37(0-41.34)$ \\
\hline
\end{tabular}

APACHE, Acute Physiology and Chronic Health Evaluation; BMI, body mass index; NT-proCNP, amino-terminal pro-C-type natriuretic peptide; SAPS, simplified acute physiology score.

Long-term follow-up data on survival were only available in 263 of 273 patients.

Significant differences between sepsis and non-sepsis patients are marked by $*(P<0.05)$ or $* *(P<0.001)$. 
plus three times the interquartile range [22]. All values, including "outliers", have been included for statistical analyzes. The Wilcoxon signed-rank test was applied as a non-parametric statistical hypothesis test for comparing repeated measurements (days one, three and seven) in the same individuals. Correlations between variables have been analysed using the Spearman correlation tests, where values of $P<0.05$ were considered statistically significant [23]. The prognostic value of the variables was tested by univariate and multivariate analyses in the Cox regression model. Kaplan-Meier curves were plotted to display the impact on survival [24]. After significant results from the univariate and multivariate Cox regression analyses, Kaplan-Meier curves and log-rank test calculations were performed subsequently for different cut-off values for NT-proCNP $(5,6,7,8,9,10,11$, and $12 \mathrm{pmol} / \mathrm{L}$ ). The threshold of $8 \mathrm{pmol} / \mathrm{L}$ yielded highest log-rank values. Receiver operating characteristic (ROC) curve analysis and the derived area under the curve (AUC) statistic provides a global and standardized appreciation of the accuracy of a marker or a composite score for predicting an event. ROC curves were generated by plotting sensitivity against 1 -specificity. All statistical analyses were performed with SPSS version 12.0 (SPSS, Chicago, IL, USA).

\section{Results}

NT-proCNP serum concentrations upon admission to the ICU are elevated in critically ill patients as compared with healthy controls

We first tested whether NT-proCNP is activated in critically ill patients. Upon admission to the medical ICU, critically ill patients displayed significantly higher NTproCNP serum concentrations as compared with healthy controls (median $0.34 \mathrm{pmol} / \mathrm{L}$, range 0 to 5.53 , in controls versus $4.07 \mathrm{pmol} / \mathrm{L}$, range 0 to 42 , in ICU patients, $P<0.001$; Figure 1a). Moreover, NT-proCNP levels were significantly higher in patients with APACHE-II scores above 10 in comparison to ICU patients admitted with APACHE-II scores of 10 or less (Figure 1b), indicating that NT-proCNP is further related to the disease severity.

\section{NT-proCNP serum concentrations indicate sepsis and organ failure in medical ICU patients}

Based on a recent study that suggested NT-proCNP as a novel biomarker for predicting sepsis in trauma patients [18], we tested whether serum NT-proCNP might identify patients with sepsis in the medical ICU setting as well. We found significantly elevated NT-proCNP serum concentrations in septic patients versus patients with

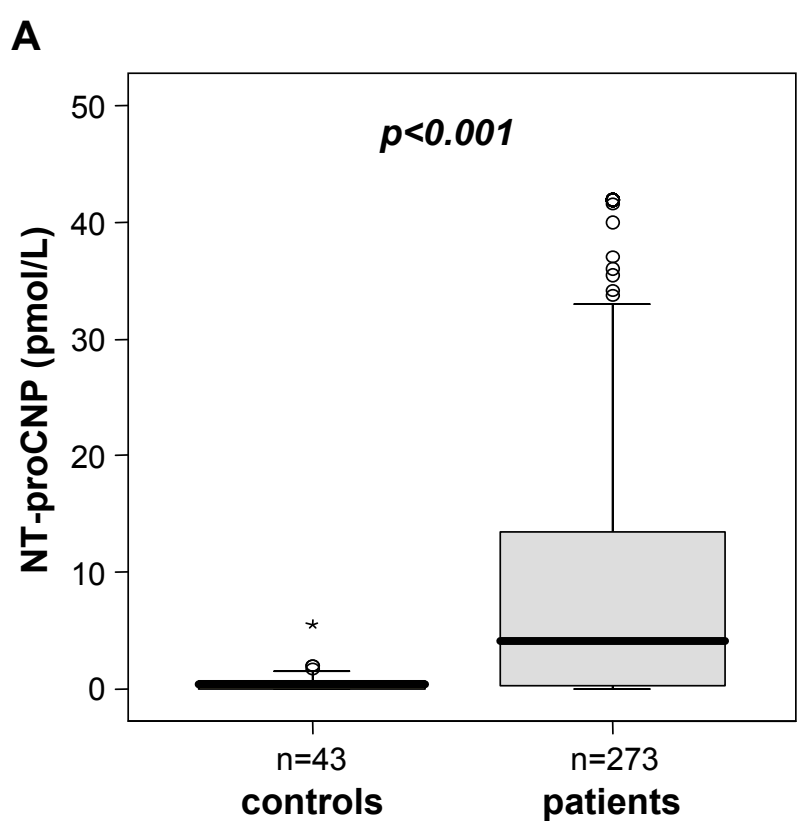

B

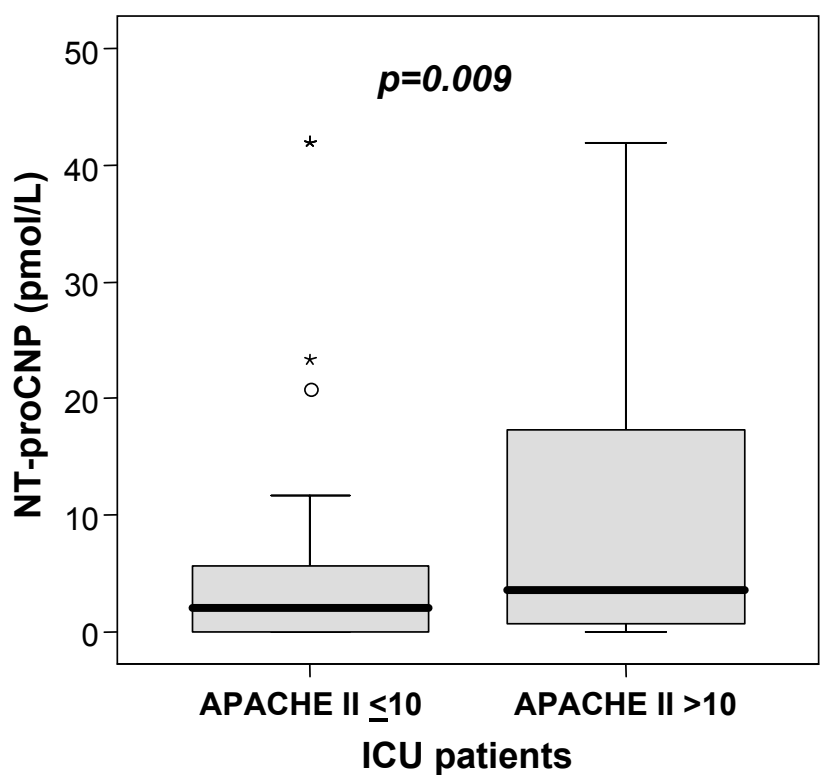

Figure 1 Serum NT-proCNP concentrations in critically ill patients at ICU admission. (a) Serum amino-terminal pro- $C$-type natriuretic peptide (NT-proCNP) concentrations at admission to the medical ICU are significantly $(P<0.001$, U-test) elevated in critically ill patients $(n=273)$ as compared with healthy controls $(n=43)$. (b) Serum NT-proCNP concentrations at admission to the medical ICU are significantly $(P=0.009$, Utest) elevated in critically ill patients with high initial Acute Physiology and Chronic Health Evaluation (APACHE) II scores (> 10) in comparison to patients with low APACHE-II scores $(</=10)$. Box plot are displayed, where the bold line indicates the median per group, the box represents $50 \%$ of the values, and horizontal lines show minimum and maximum values of the calculated non-outlier values; asterisks and open circles indicate outlier values. 
non-septic etiology of critical illness (median $1.48 \mathrm{pmol} /$ $\mathrm{L}$ in non-sepsis patients versus $5.60 \mathrm{pmol} / \mathrm{L}$ in sepsis patients; Figure 2a and Table 2). We next compared the diagnostic accuracy of NT-proCNP with classical, routinely used markers of inflammation and bacterial infection by using ROC curve analyses. C-reactive protein (CRP) and procalcitonin (PCT) achieved AUC statistics of 0.852 and 0.783 , respectively, while NT-proCNP and white blood cell count only reached AUC values of 0.661 and 0.560 , respectively (Figure $2 \mathrm{~b}$ ). Although our data demonstrated a strong elevation of NT-proCNP in critically ill patients upon admission to the ICU, NTproCNP itself evidenced inferior diagnostic accuracy for sepsis as compared with classical biomarkers.

At admission to the ICU, serum NT-proCNP concentrations in the total cohort and the subgroup of sepsis patients were closely correlated to markers of inflammation and bacterial infection, such as PCT, CRP and TNF- $\alpha$ (Table 3 ). We could also reveal strong associations with renal and hepatic functions in the total cohort of critically ill patients and in sepsis patients. In particular, we could show a close association with renal function as displayed by highly significant correlations with creatinine, urea, and cystatin $C$ serum concentrations and the glomerular filtration rate of cystatin $C$
(Table 3), representing potential renal clearance of NTproCNP in critically ill patients. Serum NT-proCNP concentrations were inversely correlated to parameters reflecting hepatic biosynthetic capacity, namely albumin (Figure 3c) and pseudocholinesterase activity (Table 3).

For the total cohort of critically ill patients, as well as for sepsis patients, we found a strong association of NTproCNP serum concentrations at admission to the ICU and established clinical scores like APACHE II, sequential organ failure assessment (SOFA) and SAPS2 (Table 3 ). These findings suggest that NT-proCNP levels are linked to disease severity in critical illness and in sepsis.

\section{NT-proCNP is a strong predictive marker for ICU and} overall survival in critically ill patients, and a decline of NT-proCNP levels after admission to the ICU is associated with a favorable outcome

Based on the clear associations between NT-proCNP, inflammatory markers, organ dysfunction and prognostic clinical scores, we hypothesized that NT-proCNP measurements could predict mortality in critically ill medical patients. We determined NT-proCNP serum concentrations at ICU admission, and at days three and seven of ICU treatment. Its prognostic impact on ICU and overall survival among all critically ill patients and the
A

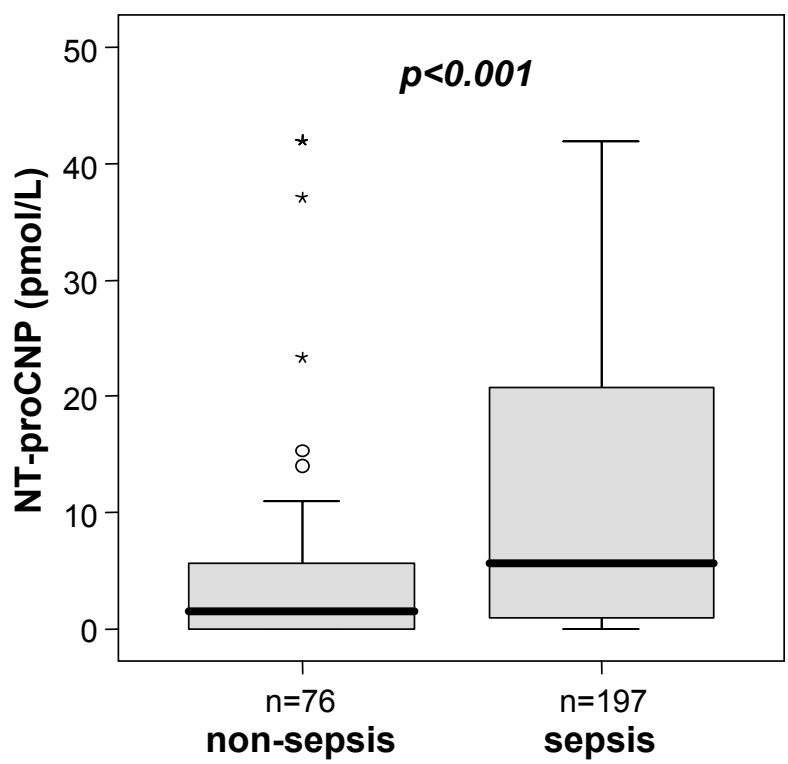

B

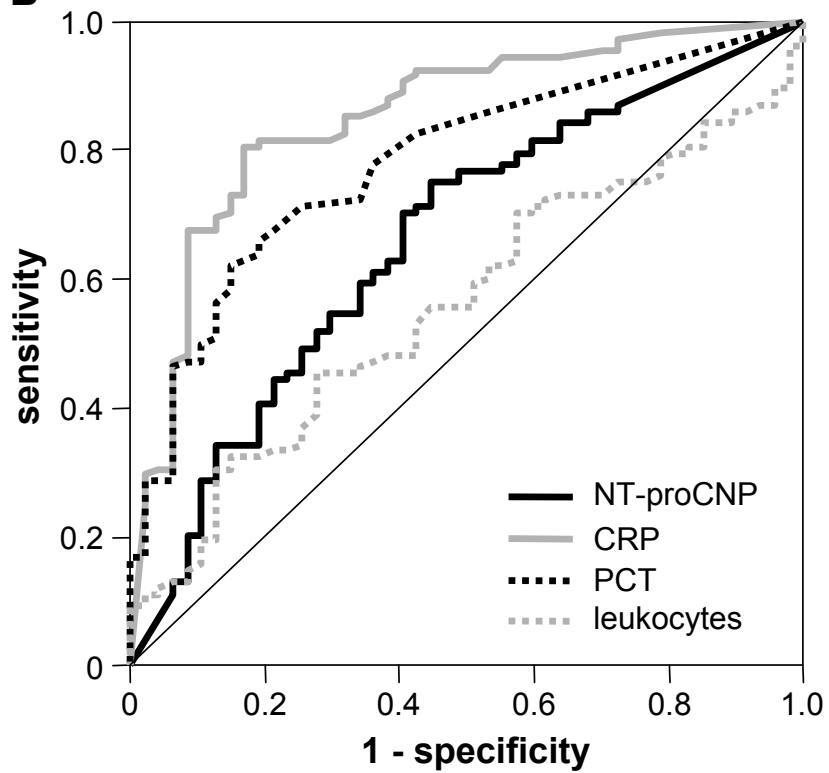

Figure 2 Serum NT-proCNP concentrations in critically ill patients are elevated in sepsis. (a) In patients with sepsis amino-terminal pro-Ctype natriuretic peptide (NT-proCNP) serum concentrations are significantly $(P<0.001$, U-test) higher as compared with patients with non-septic etiology of critical illness. Box plot are displayed, where the bold line indicates the median per group, the box represents $50 \%$ of the values, and horizontal lines show minimum and maximum values of the calculated non-outlier values; asterisks and open circles indicate outlier values. (b) Receiver operating characteristic (ROC) curve analyses comparing the diagnostic power in predicting sepsis of NT-proCNP in critically ill patients in a medical ICU (black line, area under the curve $(A \cup C)=0.661)$ with classical markers of inflammation and bacterial infection: C-reactive protein $(C R P$; grey line, $A \cup C=0.852)$, procalcitonin (PCT; dotted black line, AUC = 0.783), and white blood cell count (leucocytes; dotted grey line, AUC $=0.560$ ). 
Table 3 Correlations with NT-proCNP serum concentrations at admission

\begin{tabular}{lcccccc}
\hline & \multicolumn{2}{c}{ All patients } & \multicolumn{2}{c}{ Sepsis } & \multicolumn{2}{c}{ Non-sepsis } \\
\cline { 2 - 7 } Parameters & $\mathbf{r}$ & $\boldsymbol{P}$ & $\mathbf{r}$ & $\boldsymbol{P}$ & $\mathbf{r}$ & $\boldsymbol{P}$ \\
\hline & & & & & & \\
$\begin{array}{l}\text { Markers of } \\
\text { inflammation }\end{array}$ & & & & & & \\
$\quad$ Leukocytes & 0.178 & 0.006 & 0.196 & 0.011 & - & n.s. \\
$\quad$ CRP & 0.296 & $<0.001$ & 0.205 & 0.008 & - & n.s. \\
$\quad$ Procalcitonin & 0.456 & $<0.001$ & 0.398 & $<0.001$ & 0.316 & 0.031 \\
IL-6 & 0.188 & 0.047 & - & n.s. & - & n.s. \\
$\quad$ TNF- $\alpha$ & 0.500 & $<0.001$ & 0.444 & $<0.001$ & 0.578 & 0.001
\end{tabular}

Markers of organ function

$\begin{array}{lcccccc}\text { Creatinine } & 0.715 & <0.001 & 0.746 & <0.001 & 0.605 & <0.001 \\ \text { Urea } & 0.648 & <0.001 & 0.684 & <0.001 & 0.459 & <0.001 \\ \text { Cystatin C } & 0.700 & <0.001 & 0.757 & <0.001 & 0.419 & 0.011 \\ \text { Cystatin C GFR } & -0.707 & <0.001 & -0.749 & <0.001 & -0.749 & 0.002 \\ \text { AP } & 0.441 & 0.012 & - & \text { n.s. } & - & \text { n.s. } \\ \text { PCHE } & -0.279 & <0.001 & -0.214 & 0.009 & -0.310 & 0.015 \\ \text { Albumin } & -0.329 & <0.001 & -0.311 & 0.001 & - & \text { n.s. }\end{array}$

Clinical scores

APACHE ॥

SOFA

$0.206 \quad 0.006$

$0.261 \quad 0.026$

$0.230 \quad 0.015$

0.388

0.001

n.s.

$0.361 \quad 0.001$

SAPS2

$r$, correlation coefficient; $P, P$-value; $r$ and $P$-values by Spearman rank correlation. AP, alkaline phosphatase; APACHE, Acute Physiology and Chronic Health Evaluation; CRP, C-reactive protein; GFR, glomerular filtration rate; IL-6, interleukin 6; NT-proCNP, amino-terminal pro-C-type natriuretic peptide; PCHE, pseudocholinesterase; SAPS2, simplified acute physiology score; SOFA, sequential organ failure assessment; TNF- $\alpha$, tumor necrosis factor $\alpha$.

subgroups of sepsis and non-sepsis patients was assessed over a long-term follow-up period (median observation time 348 days, range 29 to 884 days).

Patients that died during the subsequent ICU treatment showed significantly higher NT-proCNP levels at admission and on day three (Figure $3 \mathrm{a}$ and Table 4). On day seven, a trend to higher NT-proCNP levels in patients who died in the ICU could be observed, but did not reach statistical significance. In order to account for the potential impact of volume load during ICU treatment on NT-proCNP levels, we also normalized NTproCNP concentrations to the patients' current hematocrit levels, revealing the same findings as for NTproCNP by itself (Table 5).

Moreover, low NT-proCNP levels upon admission to the ICU and on day three were a strong prognostic predictor for ICU survival (admission $P=0.001$, day three $P=$ 0.001 , day seven not significant; Cox regression analyses). In this respect, NT-proCNP levels showed comparable prognostic accuracy like established multifactorial scores such as SOFA or the SOFA score change during the first three days of ICU treatment (AUC $=0.711$ for NTproCNP, 0.684 for SOFA, and 0.646 for SOFA score changes in ROC analyses). Kaplan-Meier curves showed, using a cut-off value for serum NT-proCNP of $8 \mathrm{pmol} / \mathrm{L}$, significantly improved ICU survival for critically ill patients with low NT-proCNP levels at admission and on day three (Figures $3 \mathrm{~b}$ and 3c, and Table 4). Interestingly, survivors displayed a significant decrease in NT-proCNP serum concentrations from admission to day three $(P=0.001$; Figure 3d), while NT-proCNP levels remained stably elevated in non-survivors.

In multivariate Cox regression analyses for variables obtained at ICU admission including CRP and PCT as markers of inflammation and infection, NT-proCNP remained an independent significant prognostic parameter. However, if markers of hepatic and renal dysfunction (albumin and creatinine) were included, NTproCNP did not reach independent prognostic significance (detailed data not shown).

Although the long-term outcome of critically ill patients is certainly affected by manifold factors, we also tested whether NT-proCNP levels during the early course of ICU treatment could predict the long-term survival. Patients that will die during long-term follow up had significantly higher NT-proCNP levels than survivors at ICU admission and day three (Figure 4a). By Cox regression analyses, high NT-proCNP levels at admission $(P=0.002)$ and day three $(P=0.013)$ predicted long-term mortality in critically ill patients. We also observed a trend to high levels predicting mortality for NT-proCNP measured at day seven. Using Kaplan-Meier curves, with cut-off values for serum NT-proCNP of $8 \mathrm{pmol} / \mathrm{L}$, we demonstrated significantly improved overall survival for critically ill patients with low NT-proCNP (Figures $4 \mathrm{~b}$ and 4c; Table 4).

In an analogous manner as for short-term survival, a decrease in NT-proCNP serum concentrations from day one to three was associated with a favorable long-term prognosis. This was displayed by a significant decline in NT-proCNP levels from admission to day three in survivors $(P=0.010$, Figure $4 \mathrm{~d})$, but not in non-survivors.

\section{Discussion}

NT-proCNP has been recently proposed as a novel diagnostic marker for sepsis in traumatized patients without traumatic brain injury [18]. It was suggested that NTproCNP levels above a range of 1.6 to $3.1 \mathrm{pmol} / \mathrm{L}$ identified sepsis with high sensitivity and specificity in these patients [18]. However, the limited number of investigated patients, as well as the missing evaluation of renal function and confounding factors such as age, gender, and body mass index, have been criticized and regarded as limitations of this prior study [25]. 


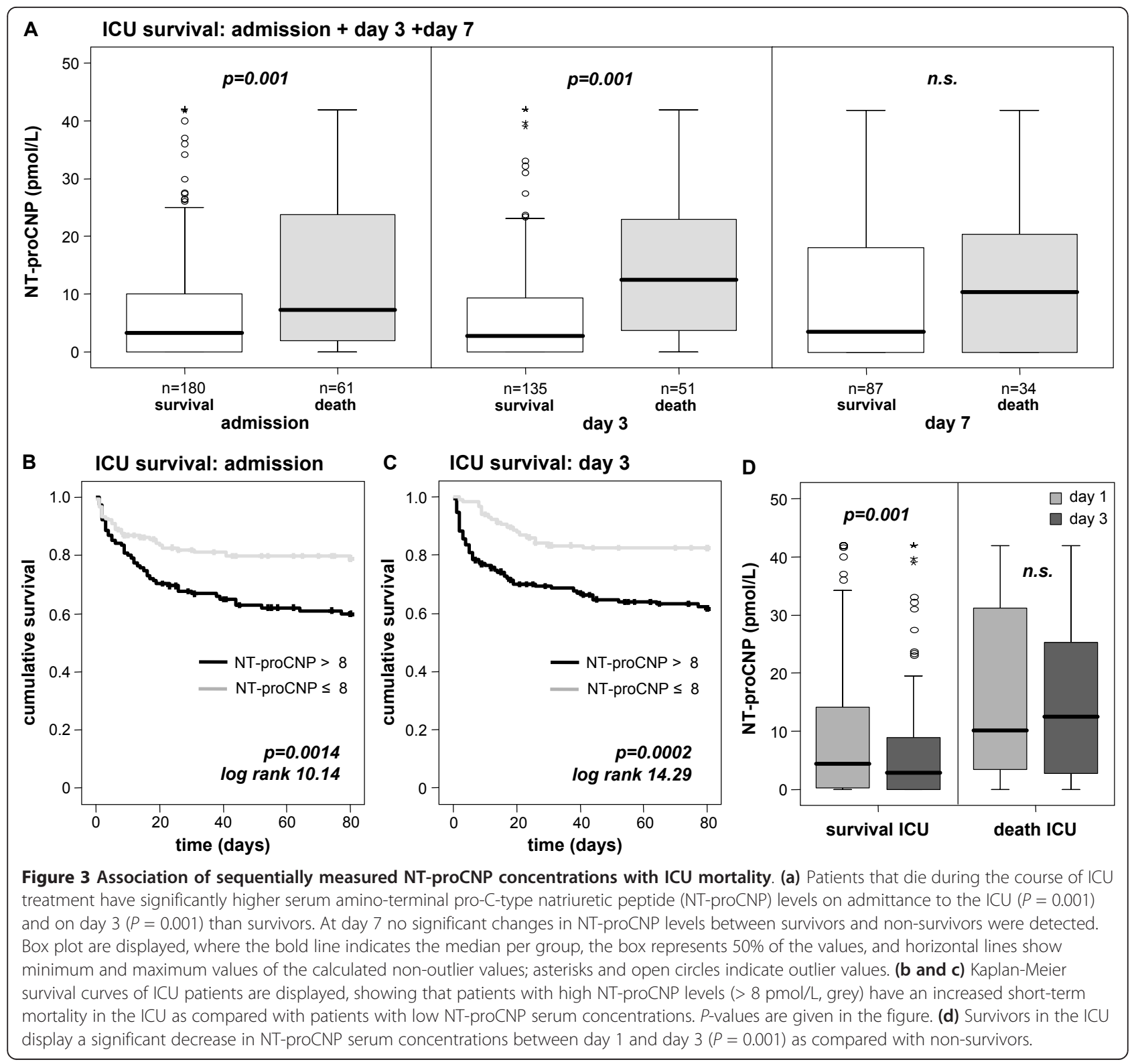

In our study, we confirmed that NT-proCNP is activated in critically ill medical patients. In accordance with previous data $[17,18]$, sepsis patients showed significantly higher NT-proCNP levels than non-sepsis patients. However, when we compared the diagnostic

Table 4 NT-proCNP serum concentrations and association with survival

\begin{tabular}{lccc}
\hline \multirow{2}{*}{ Outcome } & \multicolumn{3}{c}{ NT-proCNP } \\
\cline { 2 - 4 } & Admission & Day 3 & Day 7 \\
\hline Survivor ICU & $3.28(0-42)$ & $2.75(2.3-20)$ & $3.55(3.6-20)$ \\
Death ICU & $7.32(3.4-20)$ & $12.49(3.2-20)$ & $10.51(5.6-20)$ \\
Survivor overall & $2.53(2.31-20)$ & $2.62(2.33-20)$ & $3.32(3.67-20)$ \\
Death overall & $6.64(0-20)$ & $7.86(3.10-20)$ & $8.94(5.38-20)$ \\
\hline
\end{tabular}

NT-proCNP, amino-terminal pro-C-type natriuretic peptide. accuracy of NT-proCNP levels with routinely used biomarkers of inflammation and infection, such as CRP, PCT, and white blood cell count [26,27], NT-proCNP displayed inferior diagnostic precision for sepsis as compared with classical parameters in medical ICU patients.

Table 5 NT-proCNP serum concentrations (normalized to hematocrit) and association with survival

\begin{tabular}{lccc}
\hline \multirow{2}{*}{ Outcome } & \multicolumn{3}{c}{ NT-proCNP/hematocritmedian (range) } \\
\cline { 2 - 4 } & Admission & Day 3 & Day 7 \\
\hline Survivor ICU & $9.92(0-191)$ & $9.30(0-175)$ & $11.30(0-191)$ \\
Death ICU & $21.35(0-221)$ & $39.02(0-200)$ & $36.24(0-168)$ \\
Survivor overall & $7.67(0-191)$ & $9.04(0-175)$ & $9.51(0-175)$ \\
Death overall & $20.0(0-221)$ & $22.60(0-200)$ & $29.79(0-168)$ \\
\hline
\end{tabular}

NT-proCNP, amino-terminal pro-C-type natriuretic peptide. 


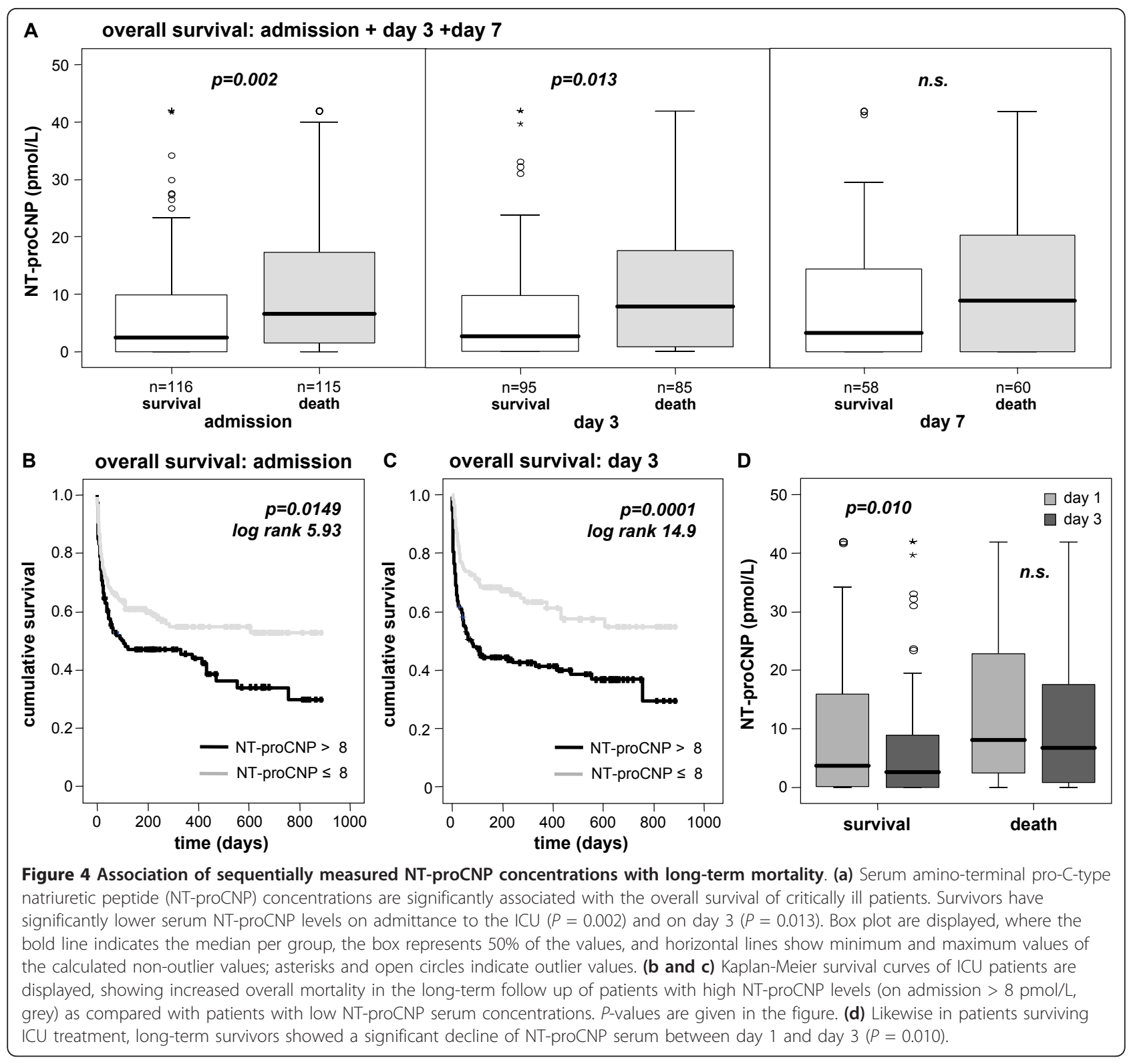

In animal models, the regulation of CNP depends on a variety of influencing factors such as glucocorticoids, thyroid hormones, vasoactive peptides, and catabolic state [28-32], but very little is known about regulatory mechanisms in states of critical illness in humans. Using correlation analyses, our study revealed significant associations between NT-proCNP and established laboratory biomarkers reflecting inflammation and organ function in medical ICU patients.

CNP itself is widely expressed in the vasculature, and highest concentrations are found in the endothelium [15]. Inflammatory cytokines such as IL-1, TNF$\alpha$, and endotoxin are known to trigger the release of CNP from endothelial cells in animal models [14], which fits well to the close correlation of serum NTproCNP with serum TNF- $\alpha$, PCT, CRP, and leukocytes in our cohort of critically ill patients. Of note, this association could not be observed for IL-6, although IL-6 serum levels were strongly increased in sepsis patients. However, we did not measure additional parameters of the $\mathrm{NO}$ and prostaglandin system such as nitrate, nitrite, prostacyclin, or thromboxane levels, because most of these parameters are rather unstable, even at $4^{\circ} \mathrm{C}$, making an appropriate pre-analytic sample acquisition in the ICU setting with a large patient cohort difficult. We therefore cannot estimate the impact of these systems on the NT-proCNP levels in critical illness. 
The pathophysiological consequences of high circulating NT-proCNP in critically ill patients are not clear at present. Very recently, CNP has also been described as a regulator of glucose metabolism by acting as an inhibitor of insulin action [33]. In a novel mouse model of inducible CNP-depletion, CNP-deficient mice were found to have reduced food intake, lower endogenous insulin levels, and a significantly reduced insulin tolerance [33]. Interestingly, NT-proCNP in critically ill patients was indeed correlated with endogenous insulin levels (C-peptide) as well as serum resistin and retinolbinding protein 4 , two mediators of insulin resistance (data not shown) [23,34]. Further studies are needed to elucidate the potential pathogenic function of NTproCNP, especially its possible involvement in promoting insulin resistance and metabolic dysregulation in the critically ill.

Our study is the first to demonstrate the prognostic value of NT-proCNP measurements in medical ICU patients. NT-proCNP serum concentrations upon admission as well as on day three were closely associated with ICU survival as well as long-term survival, and high NT-proCNP levels indicated an unfavorable prognosis. Surviving patients displayed an individual decline of NT-proCNP between days one and three. However, we would like to emphasize that the prognostic power of NT-proCNP as a biomarker is dependent on renal function as well as hepatic function, thereby limiting the general use of any static cut-off (e.g., 8 $\mathrm{pmol} / \mathrm{L}$ as shown in Kaplan-Meier curves). Larger studies should evaluate whether adjustment of NT-proCNP to individual renal and hepatic functions might further improve its diagnostic power.

\section{Conclusions}

NT-proCNP was significantly elevated in critically ill patients with highest levels in sepsis. Inflammation as well as organ function strongly impact NT-proCNP concentrations. Low NT-proCNP levels and a decline during initial treatment were associated with favorable ICU and long-term outcomes. The predictive power of serum NT-proCNP was similar to 'conventional' prognostic tools such as clinical scores. Yet, further studies are needed to elucidate the underlying pathomechanisms of NT-proCNP in patients with critical illness.

\section{Key messages}

- NT-proCNP, a paracrine molecule released mainly from vasculature, has been implicated in inflammatory and metabolic pathways and has been recently proposed as a novel biomarker for predicting sepsis in traumatized patients.

- In critically ill medical patients, NT-proCNP serum concentrations upon ICU admission are elevated as compared with healthy controls and are higher in sepsis patients than in non-sepsis patients.

- In the initial course of ICU treatment, NT-proCNP serum concentrations significantly decline between admission and day three.

- NT-proCNP levels at admission to the ICU are correlated to biomarkers of inflammation, organ dysfunction, and disease severity.

- NT-proCNP is a strong outcome predictor, and a decline of NT-proCNP serum concentrations after ICU admission is associated with a reduced mortality.

\section{Abbreviations}

ANP: atrial natriuretic peptide; APACHE: Acute Physiology and Chronic Health Evaluation; AUC: area under the curve; BNP: brain natriuretic peptide; CNP: C-type natriuretic peptide; CRP: C-reactive protein; IL: interleukin; NO: nitric oxide; NT-proCNP: amino-terminal pro-C-type natriuretic peptide; PCT: procalcitonin; ROC: receiver operating characteristic; SAPS: simplified acute physiology score; SOFA: sequential organ failure assessment; TNF-a: tumor necrosis factor $a$.

\section{Acknowledgements}

This work was supported by the German Research Foundation (DFG Ta434/ 2-1 \& SFB/TRR57). The NT-proCNP ELISA kits were a kind gift from Immundiagnostik AG, Bensheim, Germany.

\section{Authors' contributions}

$A K, F T$, and $C T$ designed the study, analyzed data and wrote the manuscript. SV performed measurements. ES, HZ, HD, and AH collected data and assisted in patient recruitment.

\section{Competing interests}

The authors declare that they have no competing interests.

Received: 2 September 2010 Revised: 6 December 2010 Accepted: 31 January 2011 Published: 31 January 2011

\section{References}

1. Nakao K, Ogawa Y, Suga S, Imura H: Molecular biology and biochemistry of the natriuretic peptide system. II: Natriuretic peptide receptors. J Hypertens 1992, 10:1111-1114.

2. Rubattu S, Sciarretta S, Valenti V, Stanzione R, Volpe M: Natriuretic peptides: an update on bioactivity, potential therapeutic use, and implication in cardiovascular diseases. Am J Hypertens 2008, 21:733-741.

3. Suttner SW, Boldt J: Natriuretic peptide system: physiology and clinical utility. Curr Opin Crit Care 2004, 10:336-341.

4. Brueckmann M, Huhle G, Lang S, Haase KK, Bertsch T, Weiss C, Kaden JJ, Putensen C, Borggrefe M, Hoffmann U: Prognostic value of plasma Nterminal pro-brain natriuretic peptide in patients with severe sepsis. Circulation 2005, 112:527-534.

5. Varpula M, Pulkki K, Karlsson S, Ruokonen E, Pettila V: Predictive value of Nterminal pro-brain natriuretic peptide in severe sepsis and septic shock. Crit Care Med 2007, 35:1277-1283.

6. Wu C, Wu F, Pan J, Morser J, Wu Q: Furin-mediated processing of Pro-Ctype natriuretic peptide. J Biol Chem 2003, 278:25847-25852.

7. Vlachopoulos C, loakeimidis N, Terentes-Printzios D, Aznaouridis K, Baou K, Bratsas A, Lazaros G, Stefanadis C: Amino-terminal pro-C-type natriuretic peptide is associated with arterial stiffness, endothelial function and early atherosclerosis. Atherosclerosis 2010, 211:649-655.

8. Sudoh T, Minamino N, Kangawa K, Matsuo H: Brain natriuretic peptide-32: $\mathrm{N}$-terminal six amino acid extended form of brain natriuretic peptide identified in porcine brain. Biochem Biophys Res Commun 1988, 155:726-732.

9. Komatsu Y, Nakao K, Suga S, Ogawa Y, Mukoyama M, Arai H, Shirakami G, Hosoda K, Nakagawa O, Hama N, et al: C-type natriuretic peptide (CNP) in rats and humans. Endocrinology 1991, 129:1104-1106. 
10. Suga S, Nakao K, Itoh H, Komatsu Y, Ogawa Y, Hama N, Imura H: Endothelial production of C-type natriuretic peptide and its marked augmentation by transforming growth factor-beta. Possible existence of "vascular natriuretic peptide system". J Clin Invest 1992, 90:1145-1149.

11. Hagiwara H, Sakaguchi H, Itakura M, Yoshimoto T, Furuya M, Tanaka S, Hirose S: Autocrine regulation of rat chondrocyte proliferation by natriuretic peptide $C$ and its receptor, natriuretic peptide receptor- $B$. J Biol Chem 1994, 269:10729-10733.

12. Schulz S: C-type natriuretic peptide and guanylyl cyclase B receptor. Peptides 2005, 26:1024-1034.

13. Scotland RS, Ahluwalia A, Hobbs AJ: C-type natriuretic peptide in vascular physiology and disease. Pharmacol Ther 2005, 105:85-93.

14. Suga S, Itoh H, Komatsu Y, Ogawa Y, Hama N, Yoshimasa T, Nakao K: Cytokine-induced C-type natriuretic peptide (CNP) secretion from vascular endothelial cells-evidence for CNP as a novel autocrine/ paracrine regulator from endothelial cells. Endocrinology 1993, 133:3038-3041.

15. Barr CS, Rhodes P, Struthers AD: C-type natriuretic peptide. Peptides 1996 17:1243-1251

16. Glauser MP, Zanetti G, Baumgartner JD, Cohen J: Septic shock: pathogenesis. Lancet 1991, 338:732-736.

17. Hama N, Itoh H, Shirakami G, Suga S, Komatsu Y, Yoshimasa T, Tanaka I, Mori K, Nakao K: Detection of C-type natriuretic peptide in human circulation and marked increase of plasma CNP level in septic shock patients. Biochem Biophys Res Commun 1994, 198:1177-1182.

18. Bahrami S, Pelinka L, Khadem A, Maitzen S, Hawa G, van Griensven M, Redl H: Circulating NT-proCNP predicts sepsis in multiple-traumatized patients without traumatic brain injury. Crit Care Med 2010, 38:161-166.

19. Gressner OA, Koch A, Sanson E, Trautwein C, Tacke F: High C5a levels are associated with increased mortality in sepsis patients-no enhancing effect by actin-free Gc-globulin. Clin Biochem 2008, 41:974-980.

20. Bone RC, Balk RA, Cerra FB, Dellinger RP, Fein AM, Knaus WA, Schein RM, Sibbald WJ: Definitions for sepsis and organ failure and guidelines for the use of innovative therapies in sepsis. The ACCP/SCCM Consensus Conference Committee. American College of Chest Physicians/Society of Critical Care Medicine. Chest 1992, 101:1644-1655.

21. Knaus WA, Draper EA, Wagner DP, Zimmerman JE: APACHE II: a severity of disease classification system. Crit Care Med 1985, 13:818-829.

22. Koch A, Sanson E, Helm A, Voigt S, Trautwein C, Tacke F: Regulation and prognostic relevance of serum ghrelin concentrations in critical illness and sepsis. Crit Care 2010, 14:R94

23. Koch A, Weiskirchen R, Sanson E, Zimmermann HW, Voigt S, Duckers H, Trautwein C, Tacke F: Circulating retinol binding protein 4 in critically ill patients before specific treatment: prognostic impact and correlation with organ function, metabolism and inflammation. Crit Care 2010, 14: R179.

24. Koch A, Weiskirchen R, Zimmermann HW, Sanson E, Trautwein C, Tacke F: Relevance of serum leptin and leptin-receptor concentrations in critically ill patients. Mediators Inflamm 2010, 2010:pii: 473540.

25. Suttner S, Boldt J: Prediction of sepsis after multiple trauma: does C-type natriuretic peptide do the trick? Crit Care Med 2010, 38:323-324

26. Giamarellos-Bourboulis EJ, Mega A, Grecka P, Scarpa N, Koratzanis G, Thomopoulos G, Giamarellou H: Procalcitonin: a marker to clearly differentiate systemic inflammatory response syndrome and sepsis in the critically ill patient? Intensive Care Med 2002, 28:1351-1356.

27. Harbarth S, Holeckova K, Froidevaux C, Pittet D, Ricou B, Grau GE, Vadas L, Pugin J: Diagnostic value of procalcitonin, interleukin-6, and interleukin-8 in critically ill patients admitted with suspected sepsis. Am J Respir Crit Care Med 2001, 164:396-402.

28. Jaszberenyi M, Bujdoso E, Telegdy G: Effects of C-type natriuretic peptide on pituitary-adrenal activation in rats. Neuroreport 1998, 9:2601-2603.

29. Prickett TC, Barrell GK, Wellby M, Yandle TG, Richards AM, Espiner EA: Response of plasma CNP forms to acute anabolic and catabolic interventions in growing lambs. Am J Physiol Endocrinol Metab 2007, 292 E1395-1400.

30. McArdle CA, Olcese J, Schmidt C, Poch A, Kratzmeier M, Middendorff R: Ctype natriuretic peptide (CNP) in the pituitary: is CNP an autocrine regulator of gonadotropes? Endocrinology 1994, 135:2794-2801.

31. Igaki T, Itoh H, Suga SI, Hama N, Ogawa Y, Komatsu Y, Yamashita J, Doi K, Chun TH, Nakao K: Effects of intravenously administered C-type natriuretic peptide in humans: comparison with atrial natriuretic peptide. Hypertens Res 1998, 21:7-13.

32. Nishimura M, Ohtsuka K, Sakamoto M, Nanbu A, Takahashi H, Yoshimura M: Roles of brain angiotensin II and C-type natriuretic peptide in deoxycorticosterone acetate-salt hypertension in rats. J Hypertens 1998, 16:1175-1185.

33. Inuzuka M, Tamura N, Yamada N, Katsuura G, Oyamada N, Taura D, Sonoyama T, Fukunaga Y, Ohinata K, Sone M, Nakao K: C-Type natriuretic peptide as a new regulator of food intake and energy expenditure. Endocrinology 2010, 151:3633-3642.

34. Koch A, Gressner OA, Sanson E, Tacke F, Trautwein C: Serum resistin levels in critically ill patients are associated with inflammation, organ dysfunction and metabolism and may predict survival of non-septic patients. Crit Care 2009, 13:R95.

doi:10.1186/cc10007

Cite this article as: Koch et al:: Prognostic value of circulating aminoterminal pro-C-type natriuretic peptide in critically ill patients. Critical Care 2011 15:R45.

\section{Submit your next manuscript to BioMed Central and take full advantage of:}

- Convenient online submission

- Thorough peer review

- No space constraints or color figure charges

- Immediate publication on acceptance

- Inclusion in PubMed, CAS, Scopus and Google Scholar

- Research which is freely available for redistribution

Submit your manuscript at www.biomedcentral.com/submit
C) Biomed Central 